\title{
A Multigrid method for restoration and regularization of images with Dirichlet boundary conditions.
}

\author{
M. Donatelli ${ }^{a}$ \\ ${ }^{a}$ Dipartimento CCFFMM, Università dell'Insubria, Via Valleggio 11, 22100 Como, Italy
}

\begin{abstract}
In many 2D image restoration problems, such as image deblurring with Dirichlet boundary conditions, we deal with two-level linear systems whose coefficient matrix is a banded block Toeplitz matrix with banded Toeplitz blocks (BTTB). Usually, these matrices are ill-conditioned since they are associated to generating functions which vanish at $(\pi, \pi)$ or in neighborhood of it. In this work, we solve such BTTB systems by applying an Algebraic Multi-Grid method (AMG). The technique we propose has an optimality property, i.e., its cost is of $O\left(n_{1} \cdot n_{2}\right)$ arithmetic operations, where $n_{1} \times n_{2}$ is the size of the images. Unfortunately, in the case of images affected by noise, our AMG method does not provide satisfactory regularization effects. Therefore, we propose two Tikhonov-like techniques, based on a regularization parameter, which can be applied to AMG method in order to reduce the noise effects.
\end{abstract}

Keywords: Point spread function, Toeplitz matrices, ill-conditioning, Multigrid methods, Tikhonov regularization.

\section{INTRODUCTION}

Basically, image restoration problems leads to the resolution of a minimization problem. If $\mathbf{g}$ denotes the observed image, then the original image $\mathbf{f}$ can be resumed by solving the minimum least square problem

$$
\min _{\mathbf{f} \in \mathbf{R}^{N}}\|K \mathbf{f}-\mathbf{g}\|_{2}
$$

where the matrix $K$ denotes the blurring operator, coming from the discretization of the Point Spread Function (PSF). Usually, the PSF is a Gaussian function endowed with a support which is much smaller than the size of the image and rapidly decreasing to zero. Since the formation of the blurred image $\mathrm{g}$ needs of data outside the area spanned by $\mathbf{f}$, the linear system $K \mathbf{f}=\mathbf{g}$ is underdetermined and hence it cannot be solved directly. To overcome this trouble, we construct a square, i.e. determined, system, by introducing certain assumptions, called boundary conditions (BCs), on the unknown boundary data of $\mathbf{f}$. In the previous literature, the widely used BCs are the Dirichlet boundary conditions (D-BCs), where a null (black) border is added outside the edges of the observed image. We remark that the D-BCs are well suited in astronomical image processing: in this case the matrix $K=T_{n, n}(z)$ is a block Toeplitz matrix with Toeplitz blocks (BTTB), usually banded at each level. The symbol $T_{n, m}(z)$ denotes a $n \times n$ blocks Toeplitz matrix with $m \times m$ Toeplitz blocks, which can be explicitly represented in the following way

$$
T_{n, m}(z)=\left(\begin{array}{cccc}
T_{0}(z) & T_{-1}(z) & \ldots & T_{1-n}(z) \\
T_{1}(z) & \ddots & \ddots & \vdots \\
\vdots & \ddots & \ddots & T_{-1}(z) \\
T_{n-1}(z) & \ldots & T_{1}(z) & T_{0}(z)
\end{array}\right)
$$

Further author information:

M. Donatelli: E-mail: marco.donatelli@uninsubria.it, Telephone: +39 0312386378, Address: Dipartimento di Chimica, Fisica e Matematica, Università dell'Insubria - Sede di Como, Via Valleggio 11, 22100 Como, Italy 


$$
T_{j}(z)=\left(\begin{array}{cccc}
a_{j, 0} & a_{j,-1} & \ldots & a_{j, 1-m} \\
a_{j, 1} & \ddots & \ddots & \vdots \\
\vdots & \ddots & \ddots & a_{j,-1} \\
a_{j, m-1} & \ldots & a_{j, 1} & a_{j, 0}
\end{array}\right), \quad j=1-n, \ldots, n-1,
$$

and such that the $a_{j, k}$ are the Fourier coefficients of the bivariate generating function $z$

$$
a_{j, k}=\frac{1}{4 \pi^{2}} \int_{\Omega} z(x, y) \mathrm{e}^{-\mathrm{i}(j x+k y)} \mathrm{d} x \mathrm{~d} y, \quad \mathrm{i}^{2}=1, \quad \Omega=[-\pi, \pi] \times[-\pi, \pi] .
$$

If the function $z$ is real and symmetric with respect to both its variables, then $T_{n, m}$ is doubly symmetric too, that is, block symmetric with symmetric blocks. We notice that the D-BCs are not the only choice. In the literature of image processing, different methods have been proposed such as, for instance, periodic boundary conditions. In this case, the matrix $K$ becomes a (block) circulant, which is usefully diagonalized by means of the discrete Fourier transform (FFT algorithm). Recently, new types of BCs namely reflective and anti-reflective BCs, have been proposed. They provide a better reconstruction than the previous popular BCs and a lower computational $\operatorname{cost}^{1,}{ }^{2}$

We underline that image restoration leads to very large linear systems, e.g. an image $512 \times 512$ leads to a linear system $2^{18} \times 2^{18}$. It is then desirable to use iterative methods to solve these systems, like Conjugate Gradient or Richardson, even suitably preconditioned. In this paper we propose a different approach, based on a Multigrid method for algebraic problems defined in. ${ }^{3}$ We stress that this technique is very useful for speeding up the convergence of any classic iterative method, and it requires of low computational costs.

We briefly discuss the computational cost in the resolution of linear systems arising in image deblurring with different BCs choices. We say that a system solvers is optimal if its total number of operations is of the same order of the matrix-vector product involved, that is, $O(N)$ in our case, being $N=n_{1} n_{2}$ the size of the images. The inversion of BTTB is known to be very expensive, e.g., the faster direct solver is of $O\left(N^{2}\right)$ operations, ${ }^{4}$ while, concerning the iterative solvers, the most popular preconditioning strategies can be far from being optimal in the polynomially ill-conditioned case. ${ }^{5}$ The discrete Fourier, cosine and sine transform require $O(N \log (N))$ complex or real operations also in the banded case. The experimental PSFs are usually generated by ill-conditioned trigonometric bivariate polynomial whose the largest degree is much lower than $n$. Therefore the corresponding matrices are ill-conditioned and (doubly) banded, that is, banded at the block level with each block banded too. The AMG we propose in this paper for BTTB is optimal also with ill-conditioned linear systems, and in the banded case it requires only $O(N)$ operations (see also ${ }^{3}$ ).

In a wide range of applications $g$ and $K$ are both corrupted by noise. In this instance, it should be stressed that the reconstruction of the original image $f$ is a very difficult task. Note indeed that the image reconstruction problem belong to the class of ill-posed problems. When the observed image is distorted, we have to adopt filtering strategies in order to reduce the noise effects, namely regularization techniques, such as the popular Tikhonov regularization method. ${ }^{6}$

In this work we customize the Tikhonov's technique because we have a linear system with a particular structure (matrix coefficients BTTB). Since the Tikhonov regularization needs of the resolution of the normal equation, we define a method for approximate the coefficient matrix $T_{n, n}^{2}(f)+\mu I_{n^{2}}$ of the normal equation, by means of the following one $T_{n, n}\left(f^{2}+\mu\right)$. Another regularization strategy was proposed by Riley. ${ }^{7}$ This approach is suitable only for symmetric and positive definite system matrices $K$, where the normal equations are not necessary to use and it is associated to a slightly different minimum problem. By an experimental point of view, both techniques give good and similar results. In the practice the regularization reduces the noise, while the AMG solves the linear system with a linear cost of $O(N)$. It follows that, it is meaningful to analyze the AMG behavior in the case of noise-free images, since we can assume that in the general case the noise is mainly removed by a regularization technique.

The outline of the paper is as follows. In section 2 we introduce the AMG for the solution of linear systems with coefficient matrix BTTB. In section 3 we study images without noise in order to show the good working (optimality) of our AMG method. A more realistic case is described in section 4, where we restore images 
corrupted by noise and where we propose two Tikhonov-like regularization techniques. At the end, in section 5 , we report some concluding remarks.

\section{ALGEBRAIC MULTIGRID FOR (MULTILEVEL) TOEPLITZ MATRICES}

Before describing the AMG used in this paper, we must introduce the $\tau$ matrix algebra, ${ }^{8}$ i.e. the space of matrices diagonalized by discrete sine transform of type I (DST I). Later, we define an AMG for $\tau$ matrices and at last we generalize this AMG to BTTB using a remarkable relationship between this two classes of matrices.

\subsection{The $\tau$ algebra (DST I)}

Let $Q_{n}$ be the $n$ dimensional DST I with entries

$$
\left[Q_{n}\right]_{i, j}=\sqrt{\frac{2}{n+1}} \sin \left(\frac{j i \pi}{n+1}\right), \quad i, j=1, \ldots, n .
$$

It is known that the matrix $Q_{n}$ is orthogonal and symmetric, i.e., $Q_{n}=Q_{n}^{T}$ and $Q_{n}^{2}=I$. Moreover, for any $n$-dimensional vector $v$, the matrix vector multiplication $Q_{n} v$ can be computed in $O(n \log n)$ real operations by fast sine transforms (FSTs). Let $\tau_{n}$ be the space containing all matrices that can be diagonalized by $Q_{n}$, i.e.,

$$
\tau_{n}=\left\{Q_{n} D_{n} Q_{n}: D_{n}=\operatorname{diag}_{j=1, \ldots, n}\left(\lambda_{j}\right)\right\}
$$

Moreover, any $A_{n} \in \tau_{n}$ can be expressed as $A_{n}=q\left(H_{n}\right)$, where $H_{n}=T_{n}(2 \cos (x))$ and $q$ is a cosine polynomial of degree at most $n-1$. It follows that, the eigenvalues $\lambda_{j}$ for $j=1, \ldots, n$ have the form $\lambda_{j}=z\left(\frac{j \pi}{n+1}\right)$, where $z(x)$ is a cosine polynomial, thus $A_{n} \in \tau_{n}$ is denoted by $A_{n}=\tau_{n}(z)$. Let $a_{j}$ be the Fourier coefficients of $z$ :

$$
a_{j}=\frac{1}{2 \pi} \int_{\pi}^{\pi} z(x) \mathrm{e}^{-\mathrm{i} j x} \mathrm{~d} x, \quad \mathrm{i}^{2}=-1, \quad j=0, \ldots, n-1,
$$

we underline the following relationship between $\tau_{n}$ and Toeplitz matrices of the same dimension:

$$
\tau_{n}(z)=\left[\begin{array}{cccc}
a_{0} & a_{1} & \cdots & a_{n-1} \\
a_{1} & \ddots & \ddots & \vdots \\
\vdots & \ddots & \ddots & a_{1} \\
a_{n-1} & \cdots & a_{1} & a_{0}
\end{array}\right]-\left[\begin{array}{ccccc}
a_{2} & \cdots & a_{n-1} & 0 & 0 \\
\vdots & . & & & 0 \\
a_{n-1} & & & & a_{n-1} \\
0 & & & . \cdot & \vdots \\
0 & 0 & a_{n-1} & \cdots & a_{2}
\end{array}\right] .
$$

Equation (4) can be expressed as $\tau_{n}(z)=T_{n}(z)-H_{n}(z)$, where $H_{n}(z)$ is the Hankel matrix on the right. We stress that when $z(x)$ is real, even and of finite order $b$, it can be expressed as

$$
z(x)=a_{0}+2 \sum_{k=1}^{b} a_{k} \cos (k x)
$$

and $H_{n}(z)$ is a low rank correction of $T_{n}(z)$ with non zero element in north-west and south-east corners. This is the case that usually happens in signal restoration, where moreover we can assume that $z(\pi)=0$ and $z(x)$ is strictly positive for all $x \in[0,2 \pi] \backslash\{\pi\}$.

Concerning the bidimensional case (e.g. images), we first mention that $\tau$ algebra has a natural version

$$
\tau_{n, n}=\left\{\left(Q_{n} \otimes Q_{n}\right) D_{n^{2}}\left(Q_{n} \otimes Q_{n}\right): D_{n^{2}}=\operatorname{diag}_{j=1, \ldots, N}\left(\lambda_{j}\right)\right\}
$$

where $Q_{n}$ is the one-level DST I transform matrix defined in (3). Moreover, in perfect analogy with the $\tau$ class in one dimension, we have that $A_{n, n} \in \tau_{n, n}$ if and only if $A_{n, n}=\tau_{n, n}(z)$ can be (uniquely) written as $\tau_{n, n}(z)=T_{n, n}(z)-H_{n, n}(z)$ that is analogous to equation (4) where each element is an one-level $\tau$ matrix. As in one dimension, matrix operations such as inversion, product, computation of the spectrum and so on can all be done by using a constant number of two-level sine transform of type I, therefore the resulting $\operatorname{cost}$ is $O(N \log n)$. When $z(x, y)$ has finite order respect each variable the matrix-vector product has a cost $O(N)$. 


\subsection{The AMG for $\tau$ matrices}

Let $A \in \mathbf{C}^{N \times N}$ be a Hermite positive definite matrix, $\mathbf{b} \in \mathbf{C}^{N}, m$ be integer with $0<m<N$. Fix integers $n_{0}=n>n_{1}>n_{2}>\cdots>n_{m}>0, N_{i}=n_{i}^{2}$ for $i=0, \ldots, m$, take $P_{i+1}^{i} \in \mathbf{C}^{N_{i+1} \times N_{i}}$ full-rank matrices and consider a class $\mathcal{R}_{i}$ of iterative methods for $N_{i}$-dimensional linear systems. The related Algebraic Multi-Grid (AMG) produces the sequence $\left\{\mathbf{x}^{(k)}\right\}_{k \in \mathbf{N}}$ according to the rule $\mathbf{x}^{(k+1)}=\mathcal{A M G}\left(0, \mathbf{x}^{(k)}, \mathbf{b}\right)$, with $\mathcal{A M G}$ recursively defined as follows:

$$
\begin{aligned}
& \mathbf{x}_{i}^{\text {(out) }}:=\mathcal{A M G}\left(i, \mathbf{x}_{i}^{(\text {in })}, \mathbf{b}_{i}\right) \\
& \text { If }(i=m) \text { Then } \operatorname{Solve}\left(A_{m} \mathbf{x}_{m}^{\text {(out) }}=\mathbf{b}_{m}\right) \\
& \text { Else } \mathbf{1} \mid \mathbf{r}_{i} \quad:=A_{i} \mathbf{x}_{i}^{(\text {in })}-\mathbf{b}_{i} \\
& 2 \quad \mathbf{b}_{i+1}:=P_{i+1}^{i} \mathbf{r}_{i} \\
& 3 \quad A_{i+1}:=P_{i+1}^{i} A_{i}\left(P_{i+1}^{i}\right)^{H} \\
& 4 \mathbf{y}_{i+1}:=\mathcal{A M G}\left(i+1, \mathbf{0}_{n_{i+1}}, \mathbf{b}_{i+1}\right) \\
& 5 \quad \mathbf{x}_{i}^{(\text {int })}:=\mathbf{x}_{i}^{(\text {in })}-\left(P_{i+1}^{i}\right)^{H} \mathbf{y}_{i+1} \\
& 6 \quad \mathbf{x}_{i}^{\text {(out) }}:=\mathcal{R}_{i}^{\nu}\left(\mathbf{x}_{i}^{\text {(int) }}\right)
\end{aligned}
$$

Step 1 calculates the residue of the proposed solution; steps $\mathbf{2}, \mathbf{3}, \mathbf{4}$ and $\mathbf{5}$ define the recursive Coarse Grid Correction (CGC) by projection $(\mathbf{2})$ of the residue, sub-grid correction $(\mathbf{3}, \mathbf{4})$ and interpolation $(\mathbf{5})$, while step 6 performs some $(\nu)$ iterations of a "post-smoother". In the definition of an AMG it is fundamental that CGC leads to a good approximation of the error in the subspace where the smoother is ineffective.

The algorithm has essentially two degrees of indetermination:

1. choice of the projectors $P_{i+1}^{i}, i=0, \ldots, m-1$;

2. choice of the smoothers $\mathcal{R}_{i}, i=0, \ldots, m-1$.

The choice of the projectors $P_{i+1}^{i}$, and the calculation of the matrices $A_{i}$ is performed before the beginning of the AMG procedure (pre-computing phase).

We analyze a special instance of the AMG algorithm (6) for $\tau$ two-level matrix, introduced in, ${ }^{3}$ where the smoother is the relaxed Richardson iteration, namely $R_{i}=I_{N_{i}}-\omega_{i} A_{i}$ ( $\omega_{i}$ is relaxing parameter). The projector is defined as $P_{i+1}^{i}=U_{i+1}^{i} \cdot \tau_{n_{i}, n_{i}}\left(p_{i}\right)$ where $p_{i}$ is a real bivariate polynomial which will be defined in $(7)$ and (8), while $U_{i+1}^{i}: \mathbf{C}^{N_{i}} \longrightarrow \mathbf{C}^{N_{i+1}}$ is the cutting operator. $U_{i+1}^{i}$ is defined as $K_{i+1}^{i} \otimes K_{i+1}^{i}$ with $K_{i+1}^{i}$ being the $\left(n_{i+1} \times n_{i}\right)$ one-level cutting matrix defined as

$$
K_{i+1}^{i}=\left[\begin{array}{ccccccccc}
0 & 1 & 0 & & & & & \\
& & 0 & 1 & 0 & & & \\
& & & & \ddots & \ddots & \ddots & & \\
& & & & & & 0 & 1 & 0
\end{array}\right]_{n_{i+1} \times n_{i}}
$$

On each step we essentially halve the size $n_{i+1}=\frac{n_{i}-1}{2}$ on each component, starting with $n_{0}=2^{k_{0}}-1$.

If the coefficient matrix is $A_{i}=\tau_{n_{i}, n_{i}}\left(z_{i}\right)$ with $z_{i}$ having a unique zero at $w_{0}=\left(x_{0}, y_{0}\right)$ of order $2 q$, the matrix $\tau_{n_{i}, n_{i}}\left(p_{i}\right)$ is chosen with $p_{i}$ such that for $w=(x, y)$

$$
\limsup _{w \rightarrow w_{0}}\left|\frac{p_{i}(\hat{w})}{z_{i}(w)}\right|<+\infty, \quad \hat{w} \in M(w), i=0, \ldots, m-1,
$$

where

$$
0<\sum_{\hat{w} \in M(w) \cup\{w\}} p_{i}^{2}(\hat{w}), \quad i=0, \ldots, m-1,
$$

with $M(w)=\{(\pi-x, y),(x, \pi-y),(\pi-x, \pi-y)\}$ being the set of the "mirror points" of $w$.

Our AMG is optimal according to the following result which is based on the theoretical tools derived in ${ }^{3}$ :

For every $i=0, \ldots, m-1$, let $A_{i}=\tau_{n_{i}, n_{i}}\left(z_{i}\right)$ with $z_{i}$ being non-negative, if 
1. the post-smoother is $R_{i}=I_{N_{i}}-\omega_{i} A_{i}$, where $0<\omega_{i}<2 /\left\|z_{i}\right\|_{\infty}$,

2. the projector $P_{i+1}^{i}=U_{i+1}^{i} \tau_{n_{i}, n_{i}}\left(p_{i}\right)$ is such that $p_{i}$ satisfies the conditions (7) and (8),

then the AMG defined in (6) is optimal.

Clearly, if we add a pre-smoother or we increase the number of smoother iteration the convergence rate of AMG can only increase. Furthermore, different iterative methods like Richardson, Jacobi, Gauss-Seidel and others have similar spectral behavior, then the choice of the smoother it is not so crucial for the AMG optimality, while the choice of the projector is much more important.

\subsection{The AMG for BTTB matrices}

We generalize the AMG previously defined for the two-level $\tau$ algebra to the two-level Toeplitz class using the two-level generalization of relation (4), which characterizes any Toeplitz matrix as its natural $\tau$ preconditioner plus a Hankel correction.

In the $1 \mathrm{D}$ case, it is possible to preserve the exact Toeplitz structure at each level without cutting much information defining the projector as

$$
P_{i+1}^{i}=K_{i+1}^{i}\{t\} T_{n_{i}}\left(p_{i}\right),
$$

where $t$ is defined again as the degree of $p_{0}$ minus 1 (we remind that the degree of $p_{i}$ is constant with respect to i), while

$$
K_{i+1}^{i}\{t\}=\left[0_{n_{i+1}-t}^{t}\left|K_{n_{i+1}-t}^{n_{i}-2 t}\right| 0_{n_{i+1}-t}^{t}\right] \in \mathbf{R}^{\left(n_{i+1}-t\right) \times n_{i}} .
$$

Where $0_{\alpha}^{\beta} \in \mathbf{R}^{\alpha \times \beta}$ is the null matrix and $K_{n_{i+1}-t}^{n_{i}-2 t} \in \mathbf{R}^{\left(n_{i+1}-t\right) \times\left(n_{i}-2 t\right)}$ is the usual cutting matrix where we put in evidence the dimensions instead of the recursion levels. We remark that, to apply recursively the AMG, we must start from dimension $n_{0}=2^{k_{0}}-1-2 t$, hence the dimension of problem at each sublevel is $n_{i}=2^{k_{0}-i}-1-2 t$. The matrix $K_{i+1}^{i}\{t\}$ is the cutting matrix that preserves the Toeplitzness at each level cutting the lowest possible level of information.

Analogously, in the 2D case, $p_{i}$ is a suitable bivariate nonnegative polynomial of partial degrees $t_{j}+1$, with $j=1,2$. Let $U_{i+1}^{i}\{t\}=K_{i+1}^{i}\left\{t_{1}\right\} \otimes K_{i+1}^{i}\left\{t_{2}\right\}$, we define $P_{i+1}^{i}=U_{i+1}^{i}\{t\} T_{n_{i}, n_{i}}\left(p_{i}\right)$. Therefore, the two-level Toeplitz matrix at AMG recursion level $i+1$ is $T_{n_{i+1}, n_{i+1}}\left(z_{i+1}\right)=P_{i+1}^{i} T_{n_{i}, n_{i}}\left(z_{i}\right)\left(P_{i+1}^{i}\right)^{H} \in \mathbf{R}^{N_{i+1} \times N_{i+1}}$ for $i=0, \ldots, m-1$, where $n_{i+1}$ is defined as in the one-level case.

Despite of its simplicity, the proposed technique is very effective since the number of elements that we neglect is reasonably low.

\section{BLURRED IMAGES WITHOUT NOISE}

Usually, the noise is reduced by regularization techniques, thus the AMG has to only solve the linear system rapidly, if possible with a linear cost in $N$ (optimal). In this section we concentrate our attention only to the resolution of the linear system, therefore we contemplate only blurred images without noise.

As already mentioned, to reconstruct the original image $\mathbf{f}$ from a blurred image $\mathbf{g}$ without noise with D-BCs leads to a linear system

$$
T_{n, n}(z) \mathbf{f}=\mathbf{g},
$$

where the blurring matrix $T_{n, n}(z)$ is created by a rapidly decreasing gaussian PSF and generated by a real function $z(x, y)$ that vanishes only in $(\pi, \pi)$ and is positive elsewhere. If the PSF is also symmetric then $z(x, y)$ is a trigonometric polynomial of finite degree. Since all $\tau$ matrices are symmetric, the AMG proposed works only for BTTB symmetric and it can solve only systems with blurring matrices from symmetric blurring function. For non symmetric blurring functions, let $s$ be the quadrantally symmetric blurring function given by $s_{i, j}=$ $\left(z_{i, j}+z_{i,-j}+z_{-i, j}+z_{-i,-j}\right) / 4$, then $T_{n, n}(s)$ can be a good preconditioner for $T_{n, n}(z)$. This strategy has a "structural" interpretation since the blurring function $z$ is replaced by its symmetrization $s$ and if $z$ is close to symmetric then $z-s$ is small and, by linearity, $T_{n, n}(z)-T_{n, n}(s)$ is small in norm: consequently it is reasonable to expect that the preconditioner $T_{n, n}(s)$ should lead to good results. 

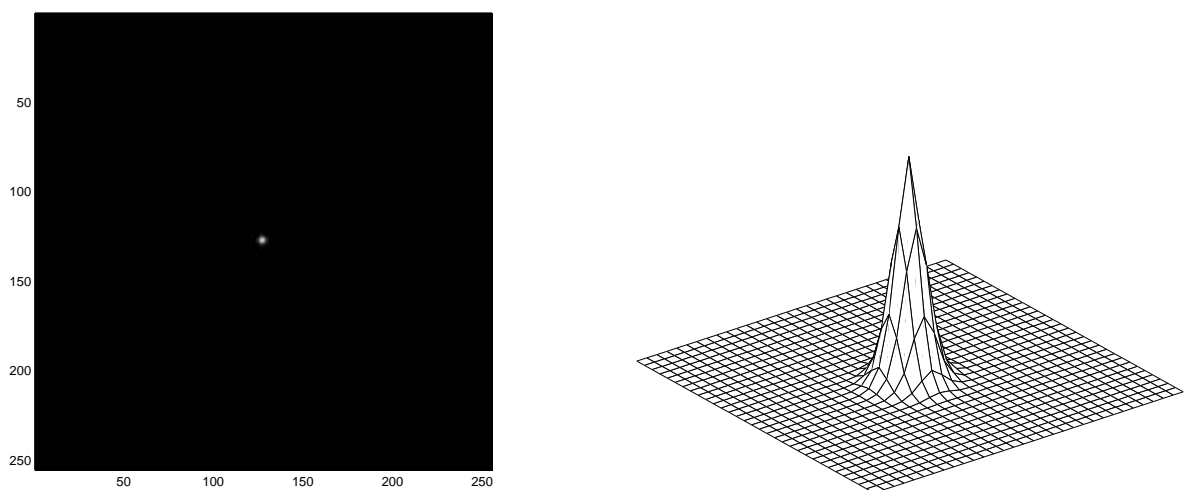

Figure 1. PSF generated by $z(x, y)$.

In the following tests we will use a PSF such that the resulting blur operator is a band approximation of the classical gaussian blur whose Fourier coefficients are positive, symmetric and decay exponentially and whose generating function is close to zero in a neighborhood of $(\pi, \pi)$ and is positive elsewhere. We define it by the "core"

$$
F(x, y)=2+\cos (x)+\cos (y)
$$

that vanishes with order two in $(\pi, \pi)$ and is positive elsewhere. The blur effect is increased multiplying $F(x, y)$ by a $\psi(x, y)>0$, obtaining a PSF with a larger support. In this way the generating function of PSF is

$$
z(x, y)=\frac{1}{c} F(x, y) \psi(x, y)
$$

where $c$ is a constant that is chosen equal to the sum of the Fourier coefficients of $F(x, y) \psi(x, y)$, so that $\left\|T_{n, n}(z)\right\|_{\infty}=1$ and the PSF calculates a weighted average of the nearest pixels, see Fig. 1 . In this way it is possible to obtain a good approximation of an experimental gaussian with a compact support. We stress that in this case the conditioning number of $T_{n, n}(z)$ is $O\left(n^{2}\right)\left(\operatorname{see}^{9}\right)$, then it is ill-conditioned.

The proposed tests are all implemented in Fortran 90 using double precision and the AMG was stopped when $\left\|\mathbf{r}^{(k)}\right\| /\|\mathbf{g}\|<10^{-7}$. The images are created with Matlab. In the AMG we do two iterations of the pre-smoother with relaxed Richardson, the relaxation parameter is $\max (z(x, y))^{-1}$, and two iterations of the post-smoother with the Conjugate Gradient without preconditioning, at each level we increase of one the number of smoother's iterations. The image that will be used in the experiments is the "satellite" developed at the US Air Force Phillips Laboratory, Lasers and Imagine Directorate, Kirtland Air Force Base, New Mexico, much used in the literature (e.g. $\mathrm{in}^{2}$ ), see Fig. 2.

In Fig. 2 the true image, the blurred image and the restored image with the proposed AMG after 48 iterations are shown in sequence; we can see that the restored image is almost equal to original image. We remark that $T_{n, n}(z)$ is ill-conditioned, but despite this bad spectral behavior, the proposed AMG is optimal as emphasized by the linear convergence reported in Fig. 3.

Finally, we remark that in the case of noise the regularized systems with $\mu>0$ ( $\mu$ is the Tikhonov parameter $\left.{ }^{10}\right)$ have a better conditioning than in the case of $\mu=0$. Therefore, our AMG, which is optimal for $\mu=0$, will be robust since the number of iterations will be bounded by a constant independent both of $N$ and of $\mu>0$.

\section{BLURRED IMAGE WITH NOISE}

We show experimentally what happens when we add some noise to the blurred image. In this case the linear system becomes

$$
T_{n, n}(z) \mathbf{f}=\mathbf{g}+\mathbf{n},
$$

where $\mathbf{n}$ is the vector that represents the noise. If the observed image is affected by noise then the latter it is amplified in the resolution of the system and the restored image results much different from the original. 


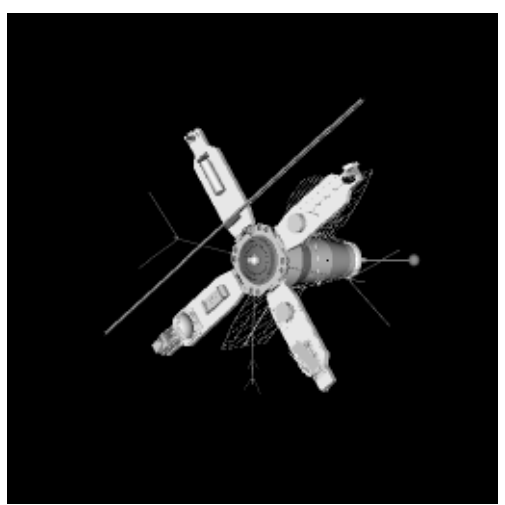

Original image (dim: $253 \times 253)$.

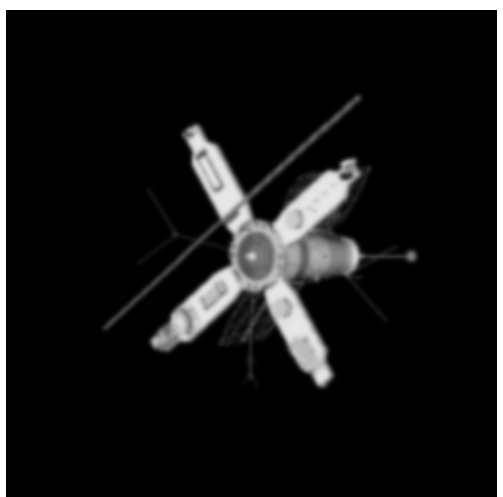

Blurred image.

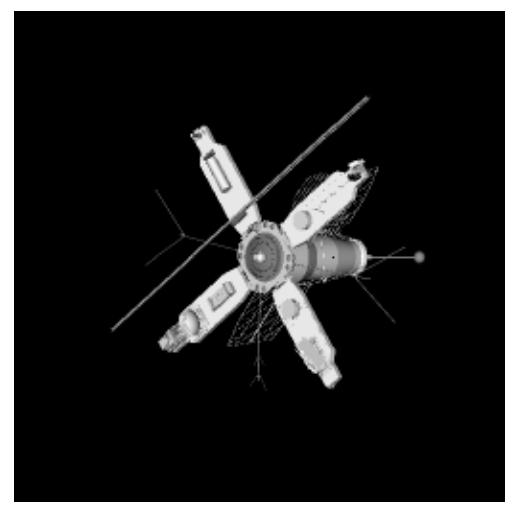

Restored image after 48 iterations

Figure 2. Sequence images of satellite.

\begin{tabular}{||c|c||}
\hline \hline \#(Iter.) & $\|$ error $\|_{2}$ \\
\hline \hline 1 & $1.533116 \mathrm{E}-01$ \\
10 & $1.110547 \mathrm{E}-02$ \\
20 & $1.373042 \mathrm{E}-03$ \\
30 & $1.813615 \mathrm{E}-04$ \\
40 & $2.440514 \mathrm{E}-05$ \\
48 & $4.928051 \mathrm{E}-06$ \\
\hline
\end{tabular}

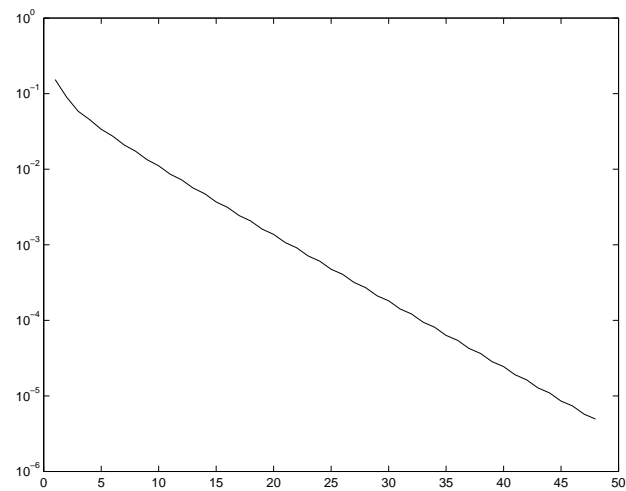

Figure 3. Relative error in $\|\cdot\|_{2}$ vs number of iterations.

According to the Toeplitz matrix theory (see e.g. ${ }^{6}$ ), if $z(\pi, \pi)=0$, then for $i$ sufficiently large the eigenvalue $\lambda_{i}$ approaches zero and is associated to a highly oscillating eigenvector (high frequency). It follows that, the inversion of a small $\lambda_{i}$ will amplify the noise coefficients and the restored image is corrupted; indeed a small percentage of noise can produce sensible variations in the restored image. The regularization technique tries to filter the high frequencies and to control the noise explosion.

In our tests the vector $\mathbf{n}$ is calculated from the noise percentage that we want to add. A percentage $\alpha$ of noise is added calculating $\mathbf{n}$ as $\mathbf{n}=\alpha \frac{\|\mathbf{g}\|_{2}}{\|\mathbf{a}\|_{2}} \mathbf{a}$, where $\mathbf{a}=\operatorname{rand}(N)$ is a vector of $N$ random components with uniform distribution.

\subsection{Restoration without regularization}

Using the technique described, we add a $2 \%$ of noise to the blurred image reported in Fig. 2 (see Fig. 5). In Fig. 4 we can see that also a little percentage of noise leads to the restored image being worse than the blurred image, according to the noise explosion previously described. Furthermore, in the same figure we can see that the relative error norm little increases at each iteration tending to a steady value. Therefore, the AMG does not have regularization properties like those of others methods, e.g. Richardson or Conjugate Gradient without preconditioning. For these methods the error first decreases and then increases when they begin to approximate the error in the high frequencies. For our AMG its optimality prevents it to be a good regularizator, since at each iteration it approximates the error in all the frequency space and not only in a subspace: therefore it is disturbed by noise at each iteration. In fact at the first level the coarse grid correction projects the problem in the subspace generated by the high frequencies and solves it also in the components corrupted by noise.

In the literature are proposed a few techniques to solve this problem, some of which are also independent of the 


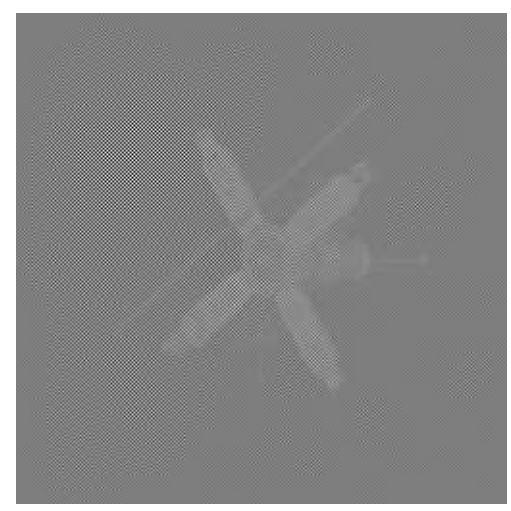

\begin{tabular}{||c|c||}
\hline \hline$\#$ (Iter.) & $\|$ error $\|_{2}$ \\
\hline \hline 1 & $1.174721 \mathrm{E}+01$ \\
10 & $1.187055 \mathrm{E}+01$ \\
20 & $1.187349 \mathrm{E}+01$ \\
30 & $1.187383 \mathrm{E}+01$ \\
40 & $1.187387 \mathrm{E}+01$ \\
51 & $1.187387 \mathrm{E}+01$ \\
\hline \hline
\end{tabular}

Relative error in $\|\cdot\|_{2}$.

Restored image after 51 iterations.

Figure 4. Restoration of the blurred satellite with noise.

method used to solve the linear system. The probably most known technique was defined by Tikhonov in ${ }^{10}$ and a specialized version of the latter will be used in the next section.

\subsection{Tikhonov-like regularization}

The regularization technique proposed by Tikhonov in $^{10}$ is based on the idea of reconstructing an image like the original and at the same time to control the noise effect. The minimum problem (1), now is reformulated as

$$
\min _{\mathbf{f} \in \mathbf{R}^{N}}\left\{\|K \mathbf{f}-\mathbf{g}\|_{2}^{2}+\mu\|\mathbf{f}\|_{2}^{2}\right\}
$$

Let $\mu=0$, then the solution of (9) is also solution of (1), but it is distorted by noise. Increasing $\mu$ we increase the weight of the part that takes under control the noise effects $\left(\|\mathbf{f}\|_{2}^{2}\right)$, but the solution calculated is a little different from the solution of (1), i.e. the restored image is a little bit modified to reduce the noise effects. Therefore, the parameter $\mu$ is a weight which allows to increase or decrease the noise distortion and the quality of the restored image. It follows that, the choice of $\mu$ is crucial to obtain a restored image as close as possible to the true image. There are many techniques to define the optimal value of $\mu$ and they are related to a theory independent of the method used to solve the linear system, thus in this paper the value of $\mu$ is determined experimentally like the value that minimizes the relative error 2 -norm.

The minimization problem (9) can be reformulated with the normal equations as

$$
\left(K^{T} K+\mu I\right) \mathbf{f}=K^{T} \mathbf{g}
$$

where $I$ is the identity matrix with the same dimensions of $K$. The numerical solution of (10) has two disadvantages:

1. the conditioning number doubles like the order of the zero if $\mu=0$,

2. the matrix $K^{T} K$ is no longer a BTTB.

We propose a technique that allows to approximate the solution $\mathbf{f}$ of (10) solving a linear system with coefficient matrix BTTB, to overcome the disadvantage (2). For the sake of notational simplicity, we first consider the $1 \mathrm{D}$ case (Toeplitz matrix) (it can be easily extended to the $2 \mathrm{D}$ case using the tensor product). Let

$$
\left(T_{n}(z) T_{n}(z)+\mu I_{n}\right) \mathbf{f}_{n}=T_{n}(z) \mathbf{g}_{n}
$$

be the linear system that we have to solve. Since $T_{n}(z)$ is symmetric, $T_{n}(z)^{2}$ is symmetric but it is not Toeplitz unless $T_{n}(z)$ is a diagonal matrix. If the bandwidth of $T_{n}(z)$ is $2 b+1$

$$
T_{n}(z)^{2}=T_{n}\left(z^{2}\right)+H_{n}\left(z^{2}\right),
$$


where $H_{n}\left(z^{2}\right)$ is a correction of rank $2 b$ with non zero entries in the north-west and south-east corners. We analyze a technique to obtain a system with a solution "near" $\mathbf{f}_{n}$ but with Toeplitz coefficient matrix. We enlarge the system (11) immersing it in a system of bigger dimension and then we apply to it a projection matrix that cuts the correction $H_{n}\left(z^{2}\right)$. The projection matrix is defined as

$$
U_{m}^{n}=\left[\begin{array}{c}
0_{b}^{n} \\
I_{n} \\
0_{b}^{n}
\end{array}\right]_{m \times n}
$$

where $m=n+2 b, 0_{b}^{n} \in \mathbf{R}^{b \times n}$ is the null matrix, therefore

$$
\left(U_{m}^{n}\right)^{T} T_{n}(z)^{2} U_{m}^{n}=T_{n}\left(z^{2}\right) .
$$

We stress that $\left(U_{m}^{n}\right)^{T} U_{m}^{n}=I_{n}$ while $U_{m}^{n}\left(U_{m}^{n}\right)^{T}$ has its first and last $b$ rows and columns of zeros while the inner part is $I_{n}$. Instead of solving the system (11) we solve the system

$$
\left(U_{m}^{n}\right)^{T}\left(T_{m}(z)^{2}+\mu I_{m}\right) U_{m}^{n}\left(U_{m}^{n}\right)^{T} \tilde{\mathbf{f}}_{m}=\left(U_{m}^{n}\right)^{T}\left[\begin{array}{c}
\bullet_{b} \\
T_{n}(z) \mathbf{g}_{n} \\
\bullet_{b}
\end{array}\right],
$$

that is the same as

$$
T_{n}\left(z^{2}+\mu\right) \tilde{\mathbf{f}}_{n}=T_{n}(z) \mathbf{g}_{n}
$$

where $\tilde{\mathbf{f}}_{n}=\left(U_{m}^{n}\right)^{T} \tilde{\mathbf{f}}_{m}$. If $U_{m}^{n}\left(U_{m}^{n}\right)^{T}=I_{m}$ then $\tilde{\mathbf{f}}_{n}=\mathbf{f}_{n}$, i.e. the solution of (12) is also solution of (11). Furthermore, for $b>0$ we have $U_{m}^{n}\left(U_{m}^{n}\right)^{T} \neq I_{m}$, but it approaches $I_{m}$ when is small. Since this is the typical case that happens in our applications, instead of solving the system (11) we solve (12) where the coefficient matrix is band Toeplitz positive-definite and therefore it is possible to use our AMG. Experimentally we can see that the solution $\tilde{\mathbf{f}}_{n}$ is a good approximation of $\mathbf{f}_{n}$.

In the $2 \mathrm{D}$ case, following the same strategy for each level, we solve

$$
T_{n, n}\left(z^{2}+\mu\right) \tilde{\mathbf{f}}_{N}=T_{n, n}(z) \mathbf{g}_{N}
$$

instead $T_{n, n}(z)^{2}+\mu I_{N} \mathbf{f}_{N}=T_{n, n}(z) \mathbf{g}_{N}$.

\subsection{Riley's regularization}

We have assumed that $z(x, y)$ is real, even in both variables and nonnegative, then $T_{n, n}(z)$ is positive definite. Therefore, our AMG can be applied without passing to the normal equations and we can apply the Tikhonov regularization according the first formulation given by Riley in $1955 .^{7}$ Instead of solving the linear system (10) we solve

$$
(K+\theta I) \mathbf{f}=\mathbf{g}
$$

which is equivalent to

$$
\min _{\mathbf{f} \in \mathbf{R}^{N}}\left\{\left\|K^{\frac{1}{2}} \mathbf{f}-K^{-\frac{1}{2}} \mathbf{g}\right\|_{2}^{2}+\theta\|\mathbf{f}\|_{2}^{2}\right\} .
$$

We underline that the factor $K^{-\frac{1}{2}} \mathbf{g}$ can cause a little amplification of the noise, but in the experimentation presented in the next subsection we can see that this method works like the Tikhonov-like method presented in the previous subsection. Usually we have $\theta>\mu$.

\subsection{Numerical experimentation in presence of regularization}

In this section we use the two techniques presented in the previous subsections to restore the blurred satellite image with a noise of $2 \%$. As already stressed, the regularized systems have a better conditioning and hence the number of iterations will be less than in the case without regularization presented in the subsection 4.1.

For the Tikhonov-like method, we solve the linear system (13), where the parameter $\mu=0.007$ is chosen as the value that minimizes the 2-norm of the relative error. In the same way, for the Riley method, we solve 


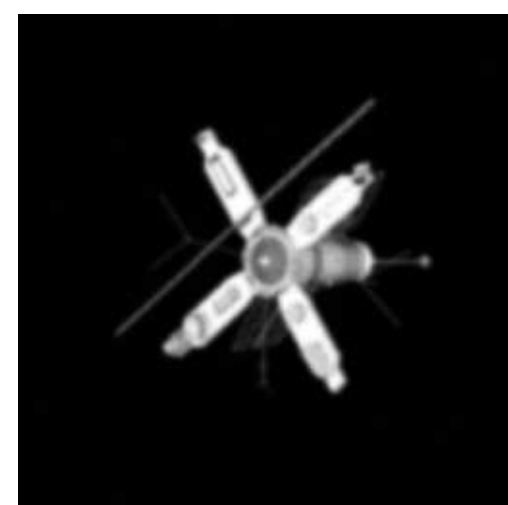

blurred image with $2 \%$ of noise.

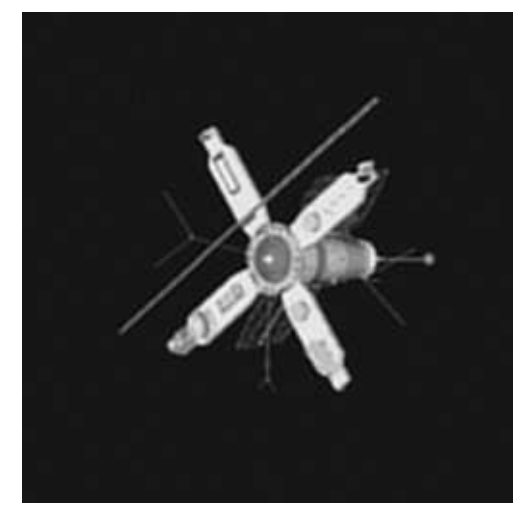

restored image with $\mathrm{AMG}+$ Tikhonov-like ( $\mu=0.007,48$ iter.).

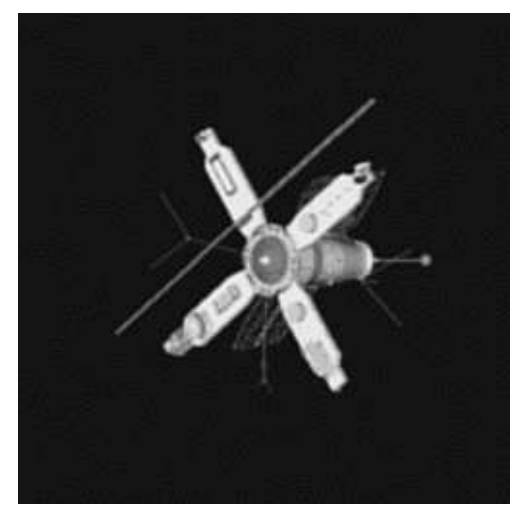

restored image with AMG + Riley $(\theta=0.07,9$ iterations).

Figure 5. Blurred image with noise and the restored image with the two proposed technique.

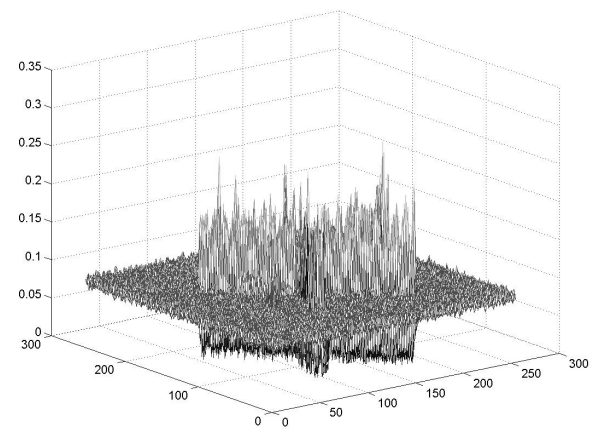

Tikhonov-like method.

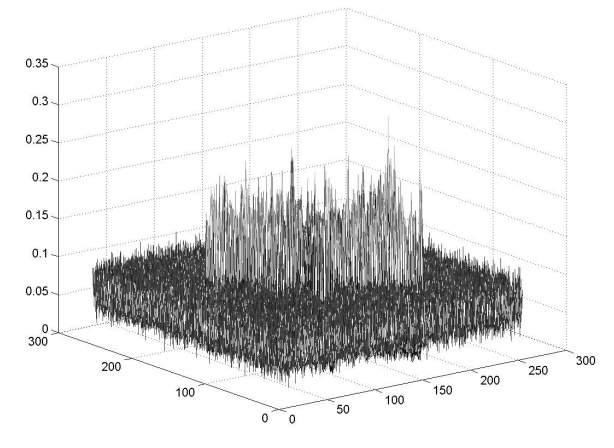

Riley method.

Figure 6. Absolute error pixel wise of the restored image

the system (14) with $\theta=0.07$. We observe that $\theta=10 \mu$, probably to reduce the noise amplification derived by $K^{-\frac{1}{2}} \mathbf{g}$. For the Riley method the generating function is bigger than the one of the Tikhonov-like method, in fact in the first case the AMG converges with 9 iterations while in the last with 48 iterations. In Fig. 5 the blurred image with noise and the two restored images are shown. As can be clearly seen from the images, we obtain similar results for both methods, but Tikhonov's has a more regular distribution of the error, obviously particularly concentrated on the satellite, as we can see by Fig. 6. Furthermore, the relative error 2-norm of the restored image is a little less with Tikhonov with respect to Riley, 0.1585 and 0.1837 respectively.

\section{CONCLUSION}

The AMG can be used to solve rapidly (optimally) the linear system, but does not have any regularization property in itself. Therefore, when the images are distorted by noise, it is necessary to use classic regularization techniques. We hope to define, in the future, a regularization technique inside the AMG.

\section{REFERENCES}

1. S. Serra Capizzano, "A note on anti-reflective boundary conditions and fast deblurring models," SIAM J. Sci. Comput. , to appear.

2. M. Ng, R. Chan, and W. C. Tang, "A fast algorithm for deblurring models with Neumann boundary conditions," SIAM J. Sci. Comput. 21(3), pp. 851-866, 1999.

3. A. Aricó, M. Donatelli, and S. Serra Capizzano, "Multigrid optimal convergence for certain (multilevel) structured linear systems," SIAM J. Matrix Anal. Appl. , submitted. 
4. N. Kalouptsidis, G. Carayannis, and D. Manolakis, "Fast algorithms for block Toeplitz matrices with Toeplitz entries," Signal Process. 6, pp. 77-81, 1984.

5. S. Serra Capizzano, "Matrix algebra preconditioners for multilevel Toeplitz matrices are not superlinear," Linear Algebra Appl. 343/344, pp. 303-319, 2002.

6. E. Tyrtyshnikov and N. Zamarashkin, "Spectra of multilevel Toeplitz matrices: advanced theory via simple matrix relationships," Linear Algebra Appl. 270, pp. 15-27, 1998.

7. J. D. Riley, "Solving systems of linear equations with a positive definite, symmetric, but possibly illconditioned matrix," Math. Tables Aids Comput. 9, pp. 96-101 English translation of Dokl. Akad. Nauk. SSSR, 151 (1963), pp. 501-504, 1955.

8. D. Bini and M. Capovani, "Spectral and computational properties of band symmetric Toeplitz matrices," Linear Algebra Appl. 52/53, pp. 99-125, 1983.

9. A. Böttcher and S. Grudsky, "On the condition numbers of large semi-definite Toeplitz matrices," Linear Algebra Appl. 279, pp. 285-301, 1998.

10. A. N. Tikhonov, "Solution of incorrectly formulated problems and regularization method," Soviet Math. Dokl. 4, pp. 1035-1038, 1963. 